\title{
Costs of care for low-energy extremity gunshot injuries are reduced with standardized treatment
}

Mai P. Nguyen MD, Jonathan C. Savakus BS, Michael S. Reich MD, Joseph F. Golob Jr. MD, Amy A. McDonald MD, John J. Como MD MPH, Heather A. Vallier MD MetroHealth Medical Center, Cleveland, Ohio, USA affiliated with Case Western Reserve University

\section{Background}

- Low-energy gunshot wounds (GSWs) are common and are associated with substantial morbidity, mortality, and socioeconomic consequences.

- Our previous works have demonstrated that a single intravenous (IV) antibiotic dose, without formal debridement in the operating room, effectively mitigates the risk of infection after extremity injury or arthrotomy secondary to low-energy GSW.1,2

- We hypothesized that the single-dose antibiotic regimen will reduce treatment expenses substantially

\section{Purposes}

To determine treatment costs associated with isolated low-energy GSWs to the extremity.

To estimate cost savings associated with a single-dose antibiotic strategy.

\section{Methods}

Institutional review board approval was obtained for the study. A series of 380 trauma patients with low-energy GSW to the
extremity from 2010-2014 was reviewed.

Comparisons of cost and treatments were performed on patients with simple GSWs treated with two different methods. Simple GSW injuries were defined as soft tissue only injuries, without intra-articular bullet fragments or acute intra articular pathology.

The protocol group was treated with single-dose antibiotics. The non-protocol group of patients were treated with deviation from the protocol with surgical debridement, prolonged antibiotic use the single dose of antibiotic prophylaxis was considered unnecessary treatment tincluding prolonged antibiotic use, admission, or surgical debridement.

Data were evaluated with two-tailed student t-tests and Fisher exact tests where appropriate. Statistical significance was set at $\alpha=0.05$.

\section{Results}

Table 1. Demographics \& injury characteristics

\begin{tabular}{|l|l|}
\hline Number of patients & 380 \\
\hline Number of GSWs & 460 or 1.2 GSWs/patient \\
\hline Age & $30.3 \pm 11.8$ years old \\
\hline Gender & $95.3 \%$ Male \\
\hline Injury Severity Score & $6.2 \pm 4.3$ \\
\hline Injury location & $78.5 \%$ lower extremities \\
\hline
\end{tabular}

Table 2. Intervention and treatments

\begin{tabular}{|l|c|}
\hline Number of admission & 309 \\
\hline Operations & 273 \\
Fracture stabilization & 126 \\
Vascular/nerve repair/ ligation & 48 \\
Irrigation and debridement & 39 \\
Others & 60 \\
\hline Days of antibiotics & 1010 \\
\hline Total cost & $\$ 1,801,554$ \\
\hline Cost per patient & $\$ 4,741$ \\
\hline Cost per GSW & $\$ 3,916$ \\
\hline
\end{tabular}

\section{Results}

- $\quad$ Analysis of 179 patients with simple GSWs who would have qualified for the single-dose antibiotic protocol without surgical debridement or admission: Table 3. Comparison between protocol groups and nonprotocol groups for simple GSWs

\begin{tabular}{|c|c|c|c|}
\hline & Protocol & Non-protocol & P values \\
\hline No of patients & 47 & 132 & \\
\hline Admissions & 0 & 108 & $<0.0001$ \\
\hline Operations & 0 & $26 I \& D$ & $<0.0002$ \\
\hline Total days of $a b x$ & 5 & 454 & 0.0002 \\
\hline Total cost & $\$ 3,619$ & $\$ 136,894$ & 0.12 \\
\hline Avg. cost per patient & $\$ 77$ & $\$ 1,037$ & $<0.0001$ \\
\hline $\begin{array}{l}\text { Infection rate } \\
\text { (only with } 90 \text { days fu) }\end{array}$ & $\begin{array}{l}18 \% \\
(8 / 44)\end{array}$ & $\begin{array}{l}10 \% \\
(3 / 30)\end{array}$ & 0.51 \\
\hline
\end{tabular}

\section{Conclusion}

Besides eliminating invasive procedures, the singledose antibiotic protocol in the emergency room can reduce treatment expenses substantially.

\section{References}

Nguyen, M. P.; Savakus, J. C.; O'Donnell, J. A.; Prayson, N. F.; Reich, M. S.; fection rates Trauma 2017; 6: 326

Nguyen MP; Reich MS; O'Donnell JA; Savakus JC; Prayson NF; Golob JF; McDonald AA; Como JJ; Vallier HA. Infection and complications after lowvelocity intra-articular gunshot injuries.J Orthop Trauma. 2017; 6: 330. 